\title{
Educação em saúde na prevenção ao câncer de mama em uma Estratégia Saúde da
}

\section{Família em Belém-PA}

\author{
Health education in the prevention of breast cancer in a Family Health Strategy in Belém-PA \\ Educación para la salud en la prevención del cáncer de mama en una Estrategia de Salud Familiar \\ en Belém-PA
}

Larissa Aline Costa Coelho

ORCID: https://orcid.org/0000-0001-9764-3579 Universidade do Estado do Pará, Brasil E-mail: larissaalineccoelho@gmail.com Liandra Silva Lopes

ORCID: https://orcid.org/0000-0001-5108-3967 Universidade do Estado do Pará, Brasil E-mail: liaa.loopes@gmail.com

Margarete Carrera Bittencourt ORCID: https://orcid.org/0000-0001-9106-0268 Universidade do Estado do Pará, Brasil

E-mail: margaretecb@gmail.com

Adriele Janaina Amorim Pereira

ORCID: https://orcid.org/0000-0002-9326-6750 Universidade do Estado do Pará, Brasil E-mail: adrielejanaina@yahoo.com.br

Tatiana Menezes Noronha Panzetti ORCID: https://orcid.org/0000-0001-7560-4595 Universidade do Estado do Pará, Brasil

E-mail: tnpanzetti@hotmail.com

Brendha Nataly Teixeira Costa

ORCID: https://orcid.org/0000-0003-3599-8336 Universidade do Estado do Pará, Brasil E-mail: brendhaenfc@gmail.com

Renata de Jesus da Silva Negrão ORCID: https://orcid.org/0000-0003-0364-0700 Universidade Federal do Pará, Brasil E-mail: renatanegrao@outlook.com.br

Flávia Savana Ribeiro de Sales ORCID: https://orcid.org/0000-0003-2409-9503 Universidade do Estado do Pará, Brasil E-mail: ssf.sales99@gmail.com Thiago Simplício Costa

ORCID: https://orcid.org/0000-0002-6349-7013 Universidade do Estado do Pará, Brasil E-mail: thiagosimplicio221@gmail.com Ilma Pastana Ferreira

ORCID: https://orcid.org/0000-0002-9152-3872 Universidade do Estado do Pará, Brasil E-mail: pastanailma@gmail.com

Stefany Ariadny Moura Braga ORCID: https://orcid.org/0000-0003-0425-2558 Universidade do Estado do Pará, Brasil E-mail: stefanyari68@gmail.com Jéssica Maria Lins da Silva

ORCID: https://orcid.org/0000-0003-3218-6447 Universidade do Estado do Pará, Brasil E-mail: jeeh.sylva@gmail.com

Valquiria Rodrigues Gomes

ORCID: https://orcid.org/0000-0003-2522-4441 Universidade do Estado do Pará, Brasil

E-mail: valquiria.rgomes@yahoo.com.br

Jéssica dos Santos Vieira

ORCID: https://orcid.org/0000-0001-5083-6429 Escola Superior da Amazônia, Brasil E-mail: jessicasv.enf.2020@gmail.com 


\title{
Resumo
}

O câncer de mama é o tumor mais prevalente entre as mulheres, sendo também a primeira causa de morte por câncer no gênero feminino, atingindo 2,1 milhões de mulheres por ano no mundo inteiro. No Brasil, prevê-se que 66.280 novos casos de câncer de mama ocorram a cada ano no triênio 2020-2022. A elevada incidência e mortalidade pelo câncer de mama está relacionada a fatores de risco. Assim, torna-se fundamental o conhecimento dos fatores de risco do câncer de mama pela educação em saúde, cujo objetivo é a promoção de saúde, como a prevenção dessa neoplasia. Dessa forma, este estudo objetivou descrever uma ação educativa voltada à prevenção do câncer de mama uma unidade de saúde. Trata-se de um estudo descritivo, com abordagem qualitativa, do tipo relato de experiência, mediante a vivência de acadêmicos de enfermagem durante uma ação educativa em saúde para uma comunidade adscrita em uma Estratégia Saúde da Família (ESF) com base no Arco de Maguerez. Como resultado, muitas mulheres inicialmente demonstravam timidez e retração, entretanto, à medida que a ação decorreu, mostraram-se mais interessadas e participativas, dando depoimentos e indagando a equipe sobre a patologia. $\mathrm{O}$ feedback de participação e envolvimento das mulheres foi massivo, uma vez que todas se engajaram nas atividades propostas. Portanto, observase que é essencial a participação de acadêmicos na comunidade, lidando diretamente com diferentes problemáticas, para o desenvolvimento de habilidades e competências necessárias à profissão, visto que este profissional trabalha constantemente com a educação em saúde dentro de sua assistência.

Palavras-chave: Câncer de mama; Prevenção; Educação em saúde.

\begin{abstract}
Breast cancer is the most prevalent tumor among women, and is also the leading cause of cancer death in women, reaching 2.1 million women per year worldwide. In Brazil, 66,280 new cases of breast cancer are expected to occur each year in the 2020-2022 period. The high incidence and mortality from breast cancer is related to risk factors. Thus, it is essential to know the risk factors for breast cancer through health education, whose objective is health promotion, such as the prevention of this neoplasm. Thus, this study aimed to describe an educational action aimed at preventing breast cancer in a health unit. This is a descriptive study, with a qualitative approach, of the experience report type, through the experience of nursing students during an educational action in health for a community included in a Family Health Strategy (ESF) based on the Arco de Maguerez. As a result, many women initially showed shyness and withdrawal, however, as the action took place, they were more interested and participative, giving testimonies and asking the team about the pathology. Feedback from the participation and involvement of women was massive, since they all engaged in the proposed activities. Therefore, it is observed that the participation of academics in the community is essential, dealing directly with different problems, for the development of skills and competences necessary for the profession, since this professional constantly works with health education within his assistance.
\end{abstract}

Keywords: Breast cancer; Prevention; Health education.

\section{Resumen}

El cáncer de mama es el tumor más prevalente entre las mujeres y también es la principal causa de muerte por cáncer en las mujeres, llegando a 2,1 millones de mujeres por año en todo el mundo. En Brasil, se espera que ocurran 66,280 nuevos casos de cáncer de mama cada año en el período 2020-2022. La alta incidencia y mortalidad por cáncer de mama está relacionada con factores de riesgo. Por ello, es fundamental conocer los factores de riesgo del cáncer de mama a través de la educación sanitaria, cuyo objetivo es la promoción de la salud, como la prevención de esta neoplasia. Así, este estudio tuvo como objetivo describir una acción educativa orientada a la prevención del cáncer de mama en una unidad de salud. Se trata de un estudio descriptivo, con abordaje cualitativo, del tipo relato de experiencia, a través de la experiencia de estudiantes de enfermería durante una acción educativa en salud para una comunidad incluida en una Estrategia de Salud de la Familia (ESF) basada en el Arco de Maguerez. Como resultado, muchas mujeres inicialmente mostraron timidez y retraimiento, sin embargo, a medida que se desarrolló la acción, se mostraron más interesadas y participativas, dando testimonios y preguntando al equipo sobre la patología. La retroalimentación de la participación e involucramiento de las mujeres fue masiva, ya que todas participaron en las actividades propuestas. Por ello, se observa que la participación de los académicos en la comunidad es fundamental, atendiendo directamente los diferentes problemas, para el desarrollo de habilidades y competencias necesarias para la profesión, ya que este profesional trabaja constantemente con la educación para la salud dentro de su asistencia.

Palabras clave: Cáncer de mama; Prevención; Educación para la salud. 


\section{Introdução}

Entre as principais causas de morbimortalidade no mundo estão as Doenças Crônicas não Transmissíveis (DNCT) e, dentre elas, há como destaque o câncer, o qual tem como característica o conjunto de comportamentos celulares anormais que estão relacionados a alterações no DNA e danos nos genes das células ao longo do tempo. Dada a relevância de sua morbimortalidade, que afeta todas as populações do mundo, sabe-se que em 2020 pouco mais de 625 mil novos casos de câncer foram previstos, e que em 2017 foram notificadas 118 mil mortes por câncer no Brasil (Carvalho, Pinto, \& Knuth, 2020; Ferreira et al., 2020).

Segundo o Instituo Nacional de Câncer - Inca (2015), o câncer de mama é definido como uma doença resultante da multiplicação desenfreada de células mamárias levando a formação de um tumor. De considerável incidência a partir dos 40 anos e aumento de até 10 vezes após os 60 anos, o carcinoma mamário é o tumor mais prevalente entre as mulheres, sendo também a primeira causa de morte por câncer para o gênero feminino, atingindo 2,1 milhões de mulheres por ano no mundo inteiro, com equivalência de 11,6\% de todos os cânceres estimados. É previsto que no Brasil 66.280 novos casos de câncer de mama ocorram a cada ano no triênio 2020-2022, o que significa um risco de 61,61 casos novos por 100 mil habitantes (Oliveira et al., 2020; Rodrigues et al., 2021; Rodrigues et al., 2020).

A elevada incidência e mortalidade pelo câncer de mama está relacionada a vários fatores de risco. O fato de ser do gênero feminino já é considerado um fator de risco para o surgimento do câncer de mama, em razão da quantidade de tecido mamário exposta ao estrogênio. Além disso, fatores como idade, obesidade e exposição ao tabaco também são considerados riscos para desenvolver essa neoplasia. De outra maneira, enfatiza-se que os fatores hereditários correspondem a somente 5\% dos casos, exceto quando o carcinoma é diagnosticado em mulheres jovens, responsável por 25\% dos casos. Portanto, a maioria das condições de risco é de origem ambiental, o que implica adotar medidas simples de prevenção de caráter primário como mudanças no estilo de vida, atividade física regular, alimentação saudável e manutenção do peso, as quais podem reduzir em 30\% os riscos dessa neoplasia (Prado et al., 2020; Santos et al., 2021).

Nesse contexto, a educação em saúde torna-se fundamental e enriquecedora ao abranger o conhecimento sobre informações dos fatores de risco do câncer de mama, tendo o intuito de promover a prevenção, estímulo ao tratamento e o aumento de sobrevida em mulheres. De outro modo, no tocante aos profissionais que desenvolvem estratégias de educação em saúde no âmbito assistencial surge o ilustre papel do enfermeiro, cujo dever é destinado ao cuidado holístico dentro da atenção básica, na ótica da proteção dos agravos de saúde, por mediar ações de promoção, prevenção e proteção à saúde (Nunes $e t$ al., 2020; Cunha et al., 2018).

Dessa forma, este estudo objetivou descrever uma ação educativa em saúde desenvolvida na Atenção Primária, em que acadêmicos de enfermagem promoveram atividades educativas voltadas à prevenção do câncer de mama como uma Estratégia de Saúde da Família com mulheres usuárias de uma Unidade de Saúde, com ampliação do conhecimento prévio acerca da temática e promoção de hábitos saudáveis para essas mulheres.

\section{Metodologia}

Este estudo configura-se como descritivo, com abordagem qualitativa, do tipo relato de experiência, efetivando-se a partir de uma vivência de acadêmicos de enfermagem durante uma ação educativa em saúde para uma comunidade adscrita em uma Estratégia Saúde da Família (ESF) de uma região periférica no município de Belém-PA.

A experiência ocorreu no mês de setembro do ano de 2019 a partir das práticas curriculares obrigatórias do curso de Enfermagem, durante o componente curricular “Enfermagem em Saúde da Mulher”, que tem como preceitos o reconhecimento das necessidades singulares das mulheres e a efetivação de uma assistência de enfermagem de maneira integral e equânime, embasados nos diversos aspectos que envolvem a saúde da mulher, bem como em uma sistematização padronizada e 
resolutiva.

Inicialmente, os discentes acompanharam consultas de enfermagem com as mulheres da unidade entre os dias de segunda a sexta-feira de uma mesma semana nos turnos da manhã, observando as necessidades do público, bem como as lacunas que poderiam ser trabalhadas no local, visando a promoção da saúde dessas mulheres. Dessa forma, evidenciou-se a partir de falas de profissionais do local, bem como de pontuações dos discentes e da docente preceptora do local, a importância de se trabalhar com a temática do câncer de mama, visto que a atenção primária é um ponto chave para trabalhar ações educativas e preventivas em saúde.

Desse modo, optou-se por utilizar a Teoria da Problematização, através do Arco de Maguerez, para a análise situacional e escolha das intervenções a serem efetuadas. Tal metodologia divide-se em cinco etapas (Figura 1), que juntas auxiliam na tomada de decisões para a escolha das ações que irão modificar uma realidade, sendo elas: observação inicial da realidade; levantamento de postos-chave; teorização com buscas na literatura científica; definição das hipóteses de solução para as problemáticas encontradas e retorno à realidade para a implementação das intervenções escolhidas (Berbel, 2011).

Figura 1 - Representação do Arco de Maguerez.

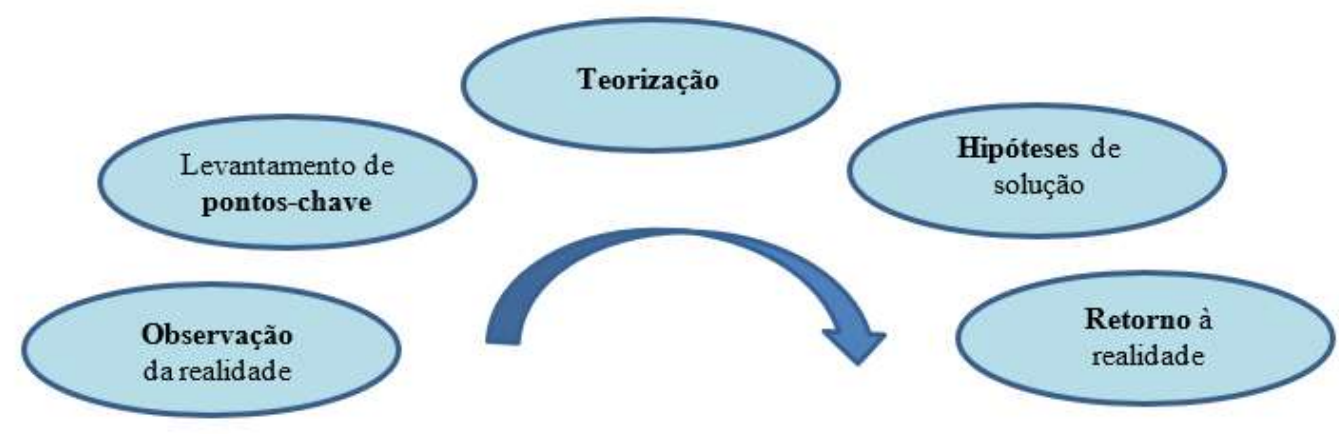

Fonte: Autores (2021).

Assim, após a observação da realidade local, levantou-se a necessidade de se trabalhar com a temática da prevenção ao câncer de mama, uma vez que foi muito pontuada pelos trabalhadores do local, reiterando a função preventiva da atenção básica. Dessa forma, efetuou-se a busca na literatura, na etapa da teorização, na qual os autores pesquisaram em bases de dados artigos que discutem a temática elegida nessa pesquisa, visando a atualização de conhecimentos para a construção da dinâmica/ação de educação em saúde. Após isso, escolheu-se como hipótese de solução o desenvolvimento de uma ação de educação em saúde, uma vez que esta permite a sensibilização do público ao passo que promove sua autonomia. Por fim, ocorreu o retorno à realidade para a aplicação das intervenções.

A ação efetivou-se em um espaço ao ar livre cedido pela unidade, com auxílio do gestor local e dos profissionais, que incentivaram a participação das mulheres do local. Ao todo houve a participação de 14 mulheres, sendo que algumas estavam acompanhadas de seus parceiros, que também assistiram as atividades. A ação desenvolveu-se nas seguintes etapas: acolhimento inicial do público com uma explicação acerca da importância do assunto abordado, roda de conversa sobre os aspectos que envolvem a patologia, bem como os mecanismos de prevenção com o uso de tecnologias leves, e uma dinâmica final para fomentar o autocuidado das participantes.

\section{Resultados}

Dentre os resultados obtidos a partir da utilização do Arco de Maguerez, evidenciou-se que este promoveu uma 
análise situacional clara, favorecendo as etapas do processo de educação em saúde, desde a escolha da temática a ser trabalhada, perpassando por sua teorização, até a efetivação da ação educativa, sendo positivo para a escolha de intervenções dentro dos serviços de saúde.

No dia da ação, houve a participação de 14 mulheres, além dos discentes, docente preceptora, gestor e profissionais do local que se dispuseram a auxiliar no decorrer das atividades. Notou-se que muitas das mulheres que estavam sendo convidadas a participar demonstravam timidez e retração, entretanto, com o incentivo dos profissionais do local, aceitaram participar e, à medida que a ação decorreu, mostraram-se mais interessadas e participativas, dando depoimentos e indagando a equipe sobre a patologia.

Durante o acolhimento inicial, ocorreu a apresentação da equipe e do público-alvo, seguido por uma explanação sobre a importância da temática. Em sequência, realizou-se uma roda de conversa na qual os acadêmicos mostraram alguns aspectos da doença, como o seu desenvolvimento, sinais e sintomas e as formas de prevenção. Nesse momento, algumas participantes indagaram acerca da sintomatologia da doença, bem como relataram casos na família de câncer de mama, reiterando a fala dos discentes sobre a importância da prevenção.

Posteriormente, durante a fala sobre a prevenção da doença, os discentes apresentaram, a partir de cartazes ilustrados, os exames de rotina que devem ser realizados por todas as mulheres, evidenciando as chances de cura a partir de um diagnóstico prévio, bem como a faixa-etária indicada para a realização de cada exame, com o auxílio de cartazes, além de demonstrar através de um molde como deve ser realizado o autoexame das mamas, reiterando que este é complementar aos outros anteriormente mencionados.

Neste ínterim, percebeu-se que muitas das partícipes repetiam os movimentos realizados, mostrando interesse na informação que estava sendo repassada. Além disso, também ocorreu o auxílio da enfermeira da unidade, que fomentava a participação e repetição dos movimentos entre as mulheres, visando a fixação deste ensinamento.

Por fim, ocorreu uma dinâmica para promover o autocuidado e a manutenção da qualidade de vida das mulheres, na qual cada participante escreveu palavras de incentivo para outra, em uma caixinha confeccionada pelos discentes, devendo ler após a finalização da atividade. Notou-se grande entrosamento e auxílio mútuo para atender a demanda solicitada, por parte das mulheres, assim como participação ativa para explicar sua frase.

O feedback de participação e envolvimento das mulheres foi massivo, uma vez que todas se engajaram a cumprir a atividade proposta. Salientou-se durante a dinâmica que a maioria das frases tinham cunho religioso, além de palavras de esperança, empoderamento, equilíbrio e bem-estar. Além disso, também houve comoção quando algumas mulheres reiteraram a importância da prevenção da doença por perdas familiares relacionadas à patologia.

Assim, houve a finalização da ação de educação em saúde, com agradecimentos por parte das mulheres que participaram, bem como do gestor e profissionais do local, que convidaram a equipe para posteriores retornos, visando trabalhar outras temáticas fundamentais dentro da atenção primária à saúde. Outrossim, releva-se a importância da educação em saúde para formação e competência de profissionais de enfermagem, visto que é um instrumento essencial na prática dos conhecimentos de autocuidado, bem como para o cuidado do outro.

\section{Discussão}

O tipo de câncer com a maior incidência e mortalidade nas mulheres de países desenvolvidos ou em desenvolvimento é o câncer de mama, sendo o segundo carcinoma mais frequente no mundo. No Brasil, a cada ano, cerca de $28 \%$ dos novos casos de câncer são correspondentes ao câncer de mama, sendo alarmante a quantidade de óbitos da doença. Se comparada a outros tipos de canceres, a taxa de incidência do câncer de mama é elevada e tem crescido, tanto pelo aumento da expectativa de vida, quanto pela adoção de hábitos de vida não saudáveis, o que reforça a necessidade de uma detecção precoce para que se 
possibilite uma prevenção precoce da doença (Batista et al., 2020; Feitosa et al., 2018; Oliveira et al., 2019; Soares et al., 2019).

O conhecimento acerca dos fatores que elevam o risco do câncer de mama é o alicerce para que se crie estratégias preventivas, o que permite um passo importante rumo à uma comunidade mais saudável. O desenvolvimento do carcinoma mamário se dá por vários fatores, sendo eles biológicos ou ambientais, de modo que entre os fatores de cunho biológico podem-se citar os endócrinos e fatores genéticos ou hereditários. Os fatores endócrinos estão relacionados ao histórico de menarca precoce, menopausa tardia, primeira gestação após os 30 anos, terapia de reposição hormonal e nuliparidade. Já quanto aos fatores ambientais estão inseridos: a exposição à radiação ionizante, sedentarismo e ingestão constante de bebidas alcoólicas (Barbosa, Ricacheneisky, \& Daudt, 2018; Gomes et al., 2020; Viegas et al., 2019).

Outrossim, quanto às medidas preventivas, pode-se citar as secundárias, as quais são mais específicas como o autoexame, avaliações regulares das mamas com profissionais da saúde e exames específicos de rotina. Dessa maneira, a informação de práticas de autocuidado contribui para efetivação do processo de educação em saúde, possibilitando o incentivo de hábitos de vida mais saudáveis, desestimulando hábitos inadequados e contemplando as necessidades de cada pessoa (Costa et al., 2020; Rocha et al., 2018).

Logo, a realização de ações de educação em saúde é essencial e necessária, visto que além de educar a população acerca da prevenção de doenças, promove a saúde, agindo como um modificador dos determinantes sociais que auxiliam nos geradores de saúde. Dentro da Atenção Básica, a qual é um conjunto de medidas voltadas à saúde no âmbito social que propõe a promoção e prevenção de doenças e seus respectivos agravos, há a Estratégia de Saúde da Família para a prevenção e detecção do câncer de mama, por meio da equipe multiprofissional, o que traz impacto positivo na saúde pública (Campos et al., 2019; Santos et al., 2021).

Nesse sentido, o enfermeiro é incumbido de gerenciar os aspectos de organização de uma unidade de saúde, realizando educação permanente, orientando e executando ações de saúde para o relacionamento pessoal, social e cultural visando integrar as necessidades da comunidade (Prado et al., 2020).

À vista disso, com os conhecimentos obtidos durante sua formação profissional, ressalta-se que o enfermeiro, além de um cuidador, é um educador. Logo, deve usufruir desses recursos práticos para a prevenção do câncer de mama e, assim, a educação em saúde mostra-se como uma prática primordial no exercício da enfermagem para prevenção e controle de agravos, além de ser uma prática imprescindível, informativa e reconhecedora do sujeito como principal agente do binômio saúdecuidado (Cunha et al., 2018; Pereira et al., 2021).

Diante desse cenário, o profissional de enfermagem, no uso dessas atribuições na atenção primária, promove assistência integral aos indivíduos e seus familiares em todas as fases da vida, sendo de responsabilidade do enfermeiro o empoderamento da mulher acerca de sua saúde e seu corpo, o que reforça a importância do enfermeiro como multiplicador de ações preventivas (Feitosa et al., 2018).

Por fim, neste âmbito, os acadêmicos de enfermagem têm uma função única ao abordar a prática educativa, mediante o desenvolvimento de habilidades e competências essenciais para o futuro da profissão, otimizando a educação e promoção em saúde através de ações de integração da comunidade no processo saúde-doença e com o olhar acolhedor e holístico, gerando impacto positivo na vida dos indivíduos (Costa et al., 2020; Pereira et al., 2021; Santos et al., 2021).

\section{Considerações Finais}

Com os objetivos atingidos, este estudo evidencia a importância de ações educativas em saúde no âmbito da atenção primária, uma vez que este nível é fundamental para a prevenção de patologias, bem como para o empoderamento da população através da sensibilização e repasse de informações de saúde, necessárias para a autonomia e autogestão eficaz dos 
indivíduos.

Além disso, evidenciou-se que o uso do Arco de Maguerez favoreceu a análise situacional, bem como a escolha da temática e da abordagem a ser utilizada, reiterando que essa metodologia auxilia diretamente na escolha de intervenções a serem efetivadas dentro dos serviços de saúde.

Para mais, salienta-se que a participação de acadêmicos dentro da comunidade, lidando diretamente com diferentes problemáticas, é essencial para o desenvolvimento de habilidades e competências necessárias à profissão, uma vez que este profissional trabalha constantemente com a educação em saúde dentro de sua assistência.

Por fim, espera-se que este trabalho fomente outras produções científicas que abordem a temática da prevenção ao câncer de mama, uma vez que essas ações são fundamentais para promover o tratamento precoce, bem como a criação de estratégias que promovam a melhora na qualidade de vida da mulher. Sugere-se para estudos futuros a realização de pesquisas envolvendo a detecção precoce, bem como os empecilhos para tal, em especial dentro da atenção básica, além de um maior número de participantes, visando maior variabilidade de resultados.

\section{Referências}

Barbosa, A. P., Ricacheneisky, L. F., \& Daudt, C. Prevenção e rastreamento de neoplasias femininas: mama e colo do útero. (2018). ACTA Médica, 39(2). https://pesquisa.bvsalud.org/portal/resource/pt/biblio-995857

Batista, G. V., Moreira, J. A., Leite, A. L., \& Moreira, C. I. H. (2020). Câncer de mama: fatores de risco e métodos de prevenção. Research, Society and Development, 9(12). http://dx.doi.org/10.33448/rsd-v9i12.11077

Berbel, N. (2011). As metodologias ativas e a promoção da autonomia de estudantes. Semina: Ciências Sociais e Humanas, 32(1), 25-40. http://www.uel.br/revistas/uel/index.php/seminasoc/article/view/10326/0

Campos, S. A. P. B. M., Almeida, C. A. P. L., Lago, E. C., Tapety, F. I., \& Alves, E. L. M. (2019). Percepções de médicos sobre a prevenção do câncer de mama. Revista de Enfermagem UFPE Online, 13(2), 315-321. https://doi.org/10.5205/1981-8963-v13i02a235056p315-321-2019

Carvalho, F. B. B., Pinto, T. J. T., \& Knuth, A. G. (2020). Atividade Física e Prevenção de Câncer: Evidências, Reflexões e Apontamentos para o Sistema Único de Saúde. Revista Brasileira de Cancerologia, 66(2). https://doi.org/10.32635/2176-9745.RBC.2020v66n2.886

Costa, P. V. D. P., Silva, J. M. L., Silva, L. C., Rocha, S. G., Costa, L. F., Soares, M. K. M., Santos, S. R. P., Lima, P. C., Rodrigues, B. N. B., Almeida, M. S., Soeiro, J. S., Martins, L. Santos, G. L., A. C. S., \& Tavares, I. I. S. (2020). A educação em saúde como ferramenta no combate ao câncer de mama: relato de experiência. Research, Society and Development, 9(10). http://dx.doi.org/10.33448/rsd-v9i10.8912

Cunha, A. R., Almeida, A. A., Fontinele, D. C. S. S., Junior, L. S. S., Oliveira, S. P. S., \& Paulino, T. S. C. (2018). O papel do enfermeiro na orientação, promoção e prevenção do câncer de mama. Revista Ser Humano - UNIFACEX, 3(1), 160-173. https://periodicos.unifacex.com.br/ humanoser/article/view/1007

Feitosa, E. M., Sá, M. A. P., Andrade, E. G. S., \& Santos, W. L. (2018). Assistência de enfermagem no rastreamento do câncer de mama. Revista JRG de Estudos Acadêmicos, 1(3). http://revistajrg.com/index.php/jrg/article/view/142/238

Ferreira, D. S., Bernardo, F. M. S., Costa, E. C., Maciel, N. S., Costa, R. L., \& Carvalho, C. M. L. (2020). Conhecimento, atitude e prática de enfermeiros na detecção do câncer de mama. Escola Anna Nery, 24(12). http://www.scielo.br/scielo.php?script=sci_arttext\&pid=S1414-81452020000200203

Gomes, K. A. L., Monteiro, L. N., Oliveira, M. E. C., Nobrega, W. F. S., Mota, G. B. C., Barbosa, D. V., \& Junior, S. A. M. (2020). Conhecimento de usuárias de um serviço público de saúde sobre fatores de risco e de proteção para o câncer de mama. Research, Society and Development, 9(9). http://dx.doi.org/10.33448/rsd-v9i9.7521

Nunes, W. L. S., Resende, W. A., Cabral, G. V. S., Oliveira, F. S. R., \& Silva, R. R. S. (2020). A importância da educação em saúde como forma de prevenção ao câncer de mama: um relato de experiência em uma unidade básica de saúde de Palmas/TO. Revista Extensão, 4(2). https://revista.unitins.br/index.php/extensao/article/view/4219

Oliveira, A. L. R., Michelin, F. S., Spada, F. C., Pires, K. G., Costa, L. O., Figueiredo, S. B. C., \& Lemos, A. P. (2019). Fatores de risco e prevenção do câncer de mama. Revista Cadernos de Medicina, 2(3), 135-145. http://www.revista.unifeso.edu.br/index.php/cadernosdemedicinaunifeso/ar ticle/view/1683\#: :text=Diversos\%20fatores\%20de\%20risco\%20tornam,maior\%20quantidade\%20de\%20tecido\%20mam\%C3\%A1rio.

Oliveira, D. A. L., Diniz, M. S. S., Silva, M. G. A., Silva, E. M., Sousa, V. J., Dutra, C. R. S., Lima, L. J. Q., Silva, M. E. S., Oliveira, M. R. P., \& Silva, J. B. (2020). Autocuidado e prevenção do câncer de mama: conhecimento das estudantes de graduação em saúde. Revista Eletrônica Acervo Saúde, 12(10), 1-8. https://doi.org/10.25248/reas.e4429.2020

Pereira, A. J. A, Silva, A. S., Oliveira, A. L. R., Brito, B. S., Lima, C. B. M., Sales, F. S. R., Tavares, I. I. S., Nascimento, J. L. M., Silva, J. M. L., Magno, J. V. E. P., Lopes, L. S., Anjos, R. B., Ramos, S. L., Mendes, S. R. C., Braga, S. A. M., \& Costa, T. S. (2021). Educação em saúde na prevenção dos agravos da hipertensão arterial: relato de experiência. Research, Society and Development, 10(3). http://dx.doi.org/10.33448/rsd-v10i3.12341 
Research, Society and Development, v. 10, n. 4, e12910413810, 2021

(CC BY 4.0) | ISSN 2525-3409 | DOI: http://dx.doi.org/10.33448/rsd-v10i4.13810

Prado, N., Loiola, P., Guimarães, T., Ohara, E. C. C., \& Oliveira, L. D. R. (2020). Gestante com diagnóstico de câncer de mama: prevenção, diagnóstico e assistência. Brazilian Journal of health Review, 3(1), 1109-1131. https://www.brazilianjournals.com/index.php/BJHR/article/view/ 6875\#: :text=O\%20c\%C3\%A2ncer\%20de\%20mama\%20associado,e\%20o\%20controle\%20do\%20c\%C3\%A2ncer.

Rocha, J. P. J., Oliveira, K. K. D., Matoso, L. M. L., Dantas, S. L. C., \& Maia, C. A. A. S. (2018). Conhecimentos de acadêmicos acerca da prevenção do câncer de colo do útero e de mama. Revista de Enfermagem da UFSM, 8(3), 464-474. https://periodicos.ufsm.br/reufsm/article/view/27839

Rodrigues, J. R. G., Salun, A. A. L. A., Oliveira, V. A. S. C., Lima, P. B., \& Nunes, M. R. (2020). Importância do enfermeiro para o controle do câncer de mama: revisão narrativa. Revista Eletrônica Acervo Saúde, (55). https://doi.org/10.25248/reas.e3668.2020

Rodrigues, F. O. S., Silveira, J. V. F., Costa, M. S., Torres, G. N., \& Faria, I. M. (2021). Amamentação na prevenção do câncer de mama: revisão de literatura. Revista Eletrônica Acervo Científico, 18, 1-8. https://doi.org/10.25248/reac.e5900.2021

Santos, C. S., Ramos, R. A. A., Santos, R. C., Pena, H. P., Oliveira, F., Almeida, C. S., Freitas, T. R., \& Andrade, S. N. (2021). Educação em saúde: prevenção do câncer de mama no Município de Divinópolis - Minas Gerais. Research, Society and Development, 10(1). http://dx.doi.org/10.33448/rsdv10i1.9465

Soares, J. C., Sousa, A. M. M., Sousa, S. M. A., Rolim, I. L. T. P. (2019). Aleitamento materno na prevenção do câncer de mama: uma revisão integrativa da literatura. Revista UNINGÁ, 56(S6), 13-22. http://revista.uninga.br/index.php/uninga/article/view/1032\#: :text=Objetivou\%2Dse\%20analisar\%20 s\%20produ\%C3\%A 7\%C3\%B5es,Virtual\%20em\%20Sa\%C3\%BAde\%20(BVS).

Viegas, A. C., Muniz, R. M., Cardoso, D. H., Santos, B. P., Machado, J. B., \& Lindemann, L. G. (2019). Prevenção do câncer de mama: conhecimento de mulheres de uma unidade saúde da família. Saúde em Revista, 19(51), 57-76. https://www.metodista.br/revistas/revistas-unimep/index.php/sr/a rticle/view/4465 